\title{
UTILIZACIÓN DE LOS SISTEMAS DE INFORMACION GEOGRAFICA PARA LA ESTIMACIÓN DE ESCORRENTÍA DIRECTA
}

\author{
USE OF GEOGRAPHIC INFORMATION SYSTEMS FOR ESTIMATED DIRECT RUNOFF
}

\author{
${ }^{1}$ Domingo M., Portuguez M.
}

\begin{abstract}
Resumen
Los Sistemas de Información Geográfica (SIG) y la hidrología son dos campos de trabajo que comparten muchos intereses. Por esa razón, cada vez más investigadores se ayudan de los SIG para la construcción de modelos hidrológicos, especialmente cuando es necesaria la representación espacial de redes de drenaje. El presente artículo examina algunos aspectos de los numerosos vínculos existentes entre los SIG y la modelización hidrológica. Estimar escorrentía a partir de información de precipitación es complejo por los diversos factores que lo controlan, como son las pendientes, el tipo de suelo y la cobertura vegetal, y más aún, cuando estos factores son tan dinámicos en el tiempo, como la cobertura, sobre todo en cuencas sometidas a una fuerte presión humana, ya que pueden alterar la producción de escorrentía. Por ello, es necesaria utilizar herramienta que facilite el cambio de caracterización de la cuenca cuando surjan dichos cambios y así determinar con más precisión la oferta hídrica. El objetivo del presente trabajo es utilizar Herramientas SIG para estimar la escorrentía directa en la cuenca del Río Guarinó, con información de los mapas temático de cobertura vegetal y uso, Suelo y Modelo Digital de Elevaciones, se genera Mapa de Numero de Curva, a todo ello se agrega data de precipitación máxima en 24 horas, como resultado se tiene los caudales máximos instantáneos para diferentes puntos de interés de la cuenca en estudio. Los resultados generados como producto de la estimación de la escorrentía directa, resultan de interés para diversas aplicaciones: estudios de impacto ambiental, ordenación territorial, manejo de cuencas y recursos naturales y predicción de riesgos.
\end{abstract}

Palabras clave: Sistemas de Información Geográfica (SIG), Modelamiento Hidrológico, modelos hidrológicos, Modelación hidrológica y SIG, Máximas avenidas.

\begin{abstract}
The Geographic Information Systems (GIS) and hydrology are two fields of work that share many interests. For that reason, more and more researchers help of GIS for construction of hydrological models, especially when the spatial representation of drainage is necessary. This article examines some aspects of the many links between GIS and hydrological modeling. Estimate runoff from precipitation information is complicated by the various factors that control it, such as slopes, soil type and vegetation cover, and even when these factors are as dynamic in time, such as coverage, especially in basins under severe human pressure, as they can alter the production of runoff. Therefore, it is necessary to use tool that facilitates the characterization change of the basin when the changes arise and determine more accurately the water supply. The aim of this work is to use GIS Tools for estimating direct runoff in the Rio Guarino, with information on thematic maps of land cover and use, soil and digital elevation model, map number curve is generated, all it adds data of maximum rainfall in 24 hours as a result one has the maximum instantaneous flows for different sites in the basin under study. The results generated as a result of the estimation of direct runoff, are of interest for various applications: environmental impact studies, land management, watershed management, natural resources and risk prediction.
\end{abstract}

Key words: Geographic Information Systems (GIS), hydrological modeling, hydrological modeling, hydrological modeling and GIS, Maxims avenues.

\section{Introduccion.}

El aprovechamiento progresivo del recurso hídrico superficial a escala temporal y espacial se ha determinado históricamente por el incremento de las demandas ligadas al abastecimiento de agua para uso humano y a las actividades industriales, de riego, agropecuarias e hidroeléctricas.

En la actualidad, cuando se habla de modelación del recurso agua, generalmente, se hace referencia a las características de modelos computacionales donde prima el nivel de experiencia conocimiento de las capacidades del software utilizado, a la cantidad y a la calidad de la información requerida, que se encuentra disponible y que ratifique la confiabilidad de los recursos obtenidos dentro de este campo.

Un tipo específico de aplicación, extensamente utilizada 
en modelación de sistemas hídricos, está representado por los programas destinados al tránsito hidrológico de eventos externos los cuales permiten conocer la distribución espacial y temporal del agua producida por una tormenta dentro de la cuenca, algún ejemplo de uso de los resultados de estos estudios, son la planificación de desarrollo sostenible de zonas especiales como páramos, así como la concepción de diseño de estructuras en ríos, tales como los gaviones, los desagües de protección, etc.

Colombia, así como el resto del mundo está influenciada por el efecto del cambio climático. Fenómenos como el del Niño o de la Niña hacen que los caudales de los ríos aumenten o disminuyan atípicamente, por consiguiente, el análisis de la sensibilidad de estas alteraciones crea la necesidad de evaluar los caudales de crecidas para poder predecir eventos catastróficos como inundaciones en la cuenca media o baja del Río Guarinó.

Sin embargo, para lograr una optimización en los resultados, el presente trabajo utiliza herramientas que en el pasado fueron manejadas de manera separada. La implementación del ArcGis, con sus extensiones ArcHydro y HECGeoHMS, para completar el análisis hidrológico con el uso del HEC HMS genera resultados que se aproxima más a la realidad.

\section{Materiales y métodos}

\section{Materiales}

\section{Información cartográfica y raster.}

La información cartográfica está compuesta por mapas temáticos elaboradas por el IGAC (Instituto Geográfico Agustín Codazzi), también se cuenta con MDE(Modelo Digital deElevación) que abarca el área de estudio y fotografías aéreas de la zona, todo estos materiales mencionados fueron proporcionados por el CIAF (Centro de Investigación y Desarrollo en Información geográfica).

Tabla 1. Información Cartográfica y raster.

\begin{tabular}{lcc}
\hline \multicolumn{1}{c}{ Información } & Estructura & Fuente \\
\hline $\begin{array}{l}\text { Mapa de Red Hídrica de la } \\
\text { cuenca }\end{array}$ & Vector & IGAC, 2007 \\
Mapa de Cobertura Vegetal & Vector & IGAC, 2007 \\
Mapa de Suelo & Vector & IGAC, 2007 \\
Modelo Digital de Elevación & Raster & IGAC, 2007 \\
Mapa de Ubicación de & Vector & IDEAM, 2007 \\
Estaciones Meteorológicas & & \\
\hline Fuente: Elaboración Propia & &
\end{tabular}

Información hidrológica: La información hidrológica fue suministrada por el IDEAM (Instituto de Hidrológica, Meteorología y estudios ambientales) en convenio con el IGAC - CIAF: Precipitaciones máximas en 24 horas.

Equipos y software: Computadora I5 2.66 Ghz DD 500 GB y 2 GB de Memoria y monitor de 17, GPS, Softwares:
Arc GIS 10, HEC - HMS 4,0, HidroEsta, extensiones: 3D Analyst, Spatial Analyst, ArcHydro Tools, HEC GeoHMS

\section{AREA DE ESTUDIO}

El área de estudio es la cuenca hidrográfica del río Guarinó con una extensión de $837.43 \mathrm{Km} 2$, Comprende sistemas geográficos de montaña y valles aluviales desde la zona de nacimiento del río a $3.100 \mathrm{msnm}$. en la vereda el Páramo del municipio de Marulanda y termina en la desembocadura del río Magdalena a $200 \mathrm{msnm}$. entre Honda y La Dorada.

Se ubica en los departamentos de Caldas, en los municipios de: La Dorada, Victoria, Marquetalia, Manzanares y Marulanda y Tolima, en los municipios de: Herveo, Fresno, Mariquita y Honda, aproximadamente el 75\% de la cuenca se localiza en el departamento de Caldas y un $25 \%$ hace parte de la jurisdicción departamental del Tolima.

\section{PROCEDIMIENTO}

Se procedió de acuerdo al Modelo Conceptual y Cartográfico, esto contempla los factores que intervienen en la predicción de la escorrentía.

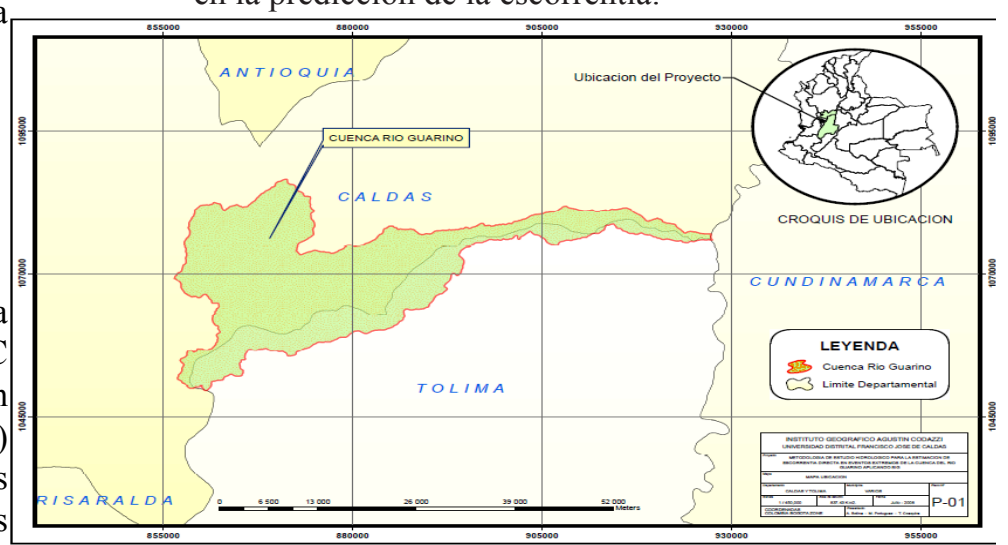

Figura 1. Mapa de ubicación de la Cuenca del río Siguas.

\section{Modelo Conceptual}

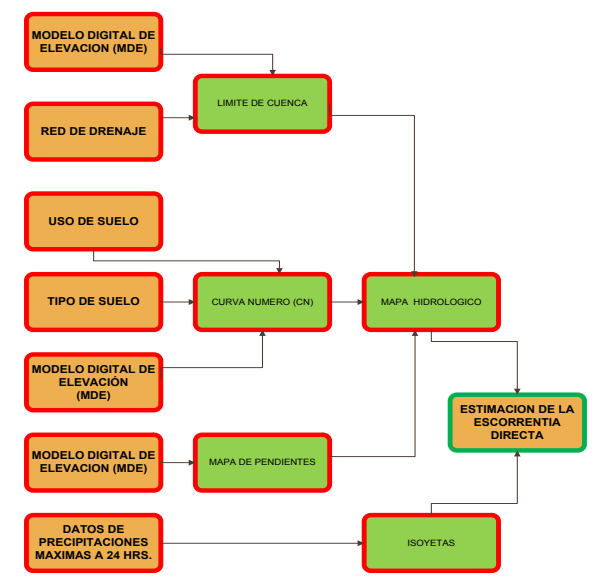

Figura 2. Modelo conceptual para estimar la escorrentía directa. Fuente: Elaboración Propia 


\section{Modelo cartográfico}

Modelo de secuencia de procedimientos relacionados con las operaciones que incluyen superposiciones, manipulación de datos y conversiones de formato.

Este modelo está dividido por las extensiones de ArcHydro Tools, Hec-GeoHMS y por el programa HMS, Ver fig. 3.

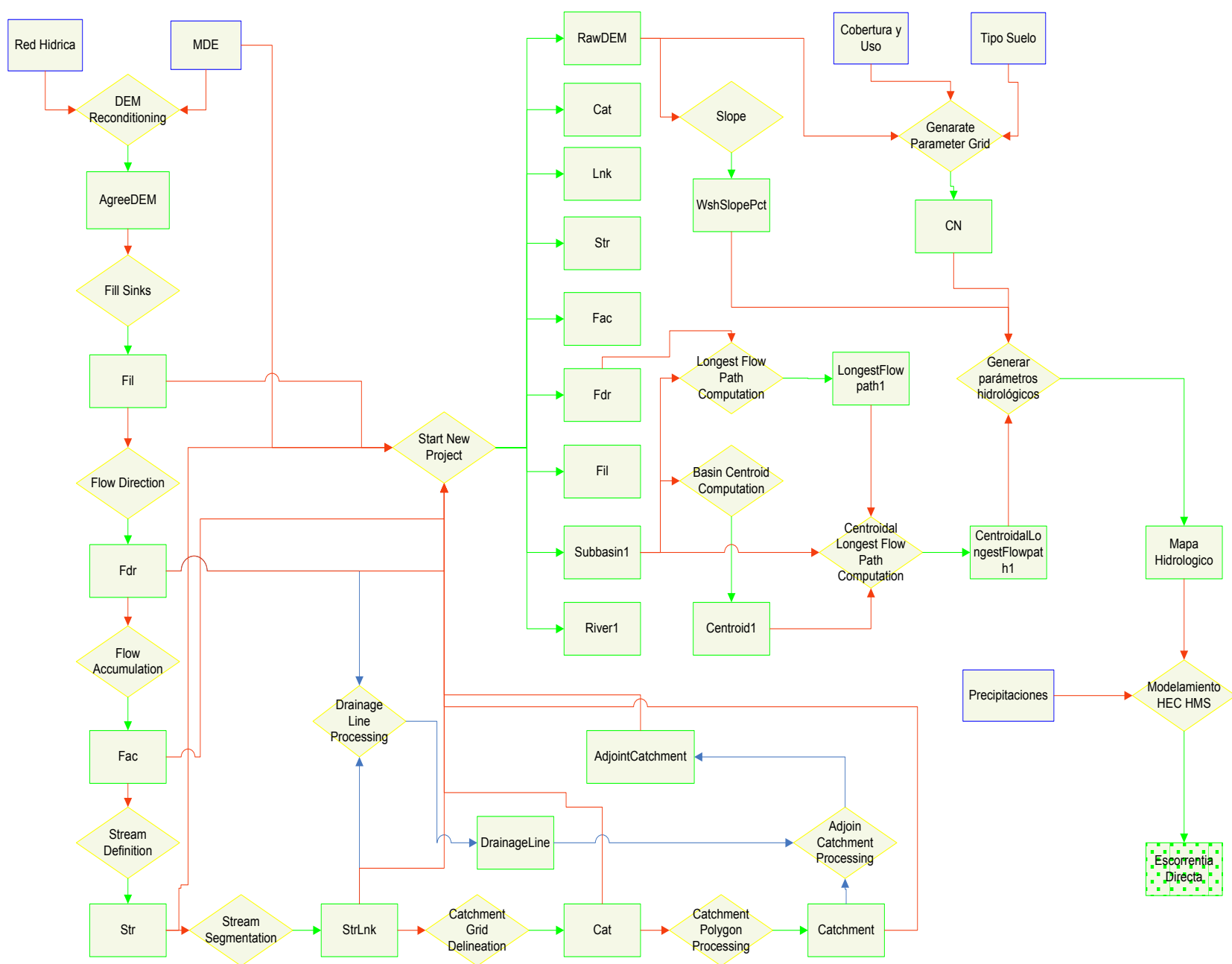

Figura 3. Modelo cartográfico para estimar la escorrentía directa.

Fuente: Elaboración Propia

\section{Determinación de parámetros.}

\section{a. Métodos de abstracción de precipitación}

Las abstracciones hidrológicas son los procesos físicos que actúan para reducir la precipitación total a precipitación efectiva. La precipitación efectiva constituye el escurrimiento de superficie, para el cálculo de tasas de pérdidas se utilizó el método de Soil Conservation Service.

\section{b.Transformación Precipitación - Escorrentía}

Para la transformación de la lluvia en escurrimiento se utilizó el método de hidrográma SCS, para lo requiere el SCSLag, (también llamado tiempo de retardo) calculándose de la siguiente manera ${ }^{2}$ :

$$
\begin{gathered}
\text { SCS Lag }(h r)=0.6 * T_{c}(h r) \\
T_{c}(h r)=0.3\left(\frac{L}{S^{0.25}}\right)^{0.77}
\end{gathered}
$$

Dónde:

$\mathrm{L}=$ Longitud del cauce $(\mathrm{km})$

$\mathrm{S}=$ Pendiente media $(\mathrm{m} / \mathrm{m})$

$\mathrm{Tc}=$ Tiempo de concentración (hr).

\section{c. Transito hidrológico en cauce}

El programa HEC-HMS permite escoger entre los siguientes modelos a la hora de tratar de representar la transformación que experimenta el hidrográma entre los puntos inicial y final de un tramo de cauce, para ello se utilizó el Modelo de Onda Cinemática.

En el HEC HMS se requiere los siguientes datos:

$\mathrm{n}=$ Coeficiente de rugosidad de manning

$\mathrm{w}=$ Ancho de cauce

$\mathrm{xH}: 1 \mathrm{~V}=$ valor para distancia horizontal 


\section{d. Análisis de frecuencia para una variable hidrológica (precipitación)}

El análisis de frecuencia es una herramienta utilizada para, predecir el comportamiento futuro de los caudales en un sitio de interés, a partir de la información histórica de caudales. Es un método basado en procedimientos estadísticos que permite calcular la magnitud del caudal asociado a un período de retorno. $\mathrm{Su}$ confiabilidad depende de la longitud y calidad de la serie histórica, además de la incertidumbre propia de la distribución de probabilidades seleccionada. Cuando se pretende realizar extrapolaciones, período de retorno mayor que la longitud de la serie disponible, el error relativo asociado a la distribución de probabilidades utilizada es más importante, mientras que en interpolaciones la incertidumbre está asociada principalmente a la calidad de los datos a modelar; en ambos casos la incertidumbre es alta dependiendo de la cantidad de datos disponibles (Ashkar, et al. 1994).

El análisis de frecuencia consiste en determinar los parámetros de las distribuciones de probabilidad y determinar con el factor de frecuencia la magnitud del evento para un período de retorno dado.

\section{e. Prueba de bondad de ajuste para las diferentes estaciones.}

La prueba compara la distribución de frecuencia observada (Fo) de una variable, con la distribución de frecuencia de la misma variable medida en un grupo de referencia.

El procedimiento de la prueba implica el cálculo de una distribución esperada (Fe) en el grupo estudiado, usando como punto de partida a la distribución de la variable en el grupo de referencia.
El 'propósito de la prueba es averiguar si existen diferencias estadísticamente significativa entre la distribución observada (Fo) y la distribución esperada $(\mathrm{Fe})$.

\section{f. Mapas de Isoyetas.}

Son isolíneas que une los puntos, en un plano cartográfico, que presentan la misma precipitación en la unidad de tiempo considerada. Así, para una misma área, se puede diseñar un gran número de mapas de isoyetas; ejemplos, isoyetas de precipitación media de largo periodo del mes de enero, febrero, etc., o las isoyetas de precipitaciones anuales.

\section{Resultados y discusión}

\section{a. Procesamiento con herramienta SIG}

Con Herramienta SIG, la cuenca quedo dividida en subcuencas, se determinaron los parámetros: centroide de cada subcuencas, área, pendiente, abstracción inicial (Ia), Número de Curva y tiempo de retardo (TLag), ver Tabla 2.

Así mismo, para tramo de rio donde se realizaría tránsito de cauce, se determinaron la longitud, pendiente, coeficiente de rugosidad de manning, tipo de sección, ancho y talud.

\section{b. Análisis de frecuencia de las precipitaciones}

Para el análisis de frecuencia se utilizaron las siguientes funciones de distribución: Distribución Normal, Distribución LogNormal 2P, Distribución Log Pearson III, Distribución de valor extremo (Gumbel)

En las tablas 4, 5, 6, 7 y 8, se muestra los resultados, para cada estación

Tabla 2. Parámetros para Subcuenca.

\begin{tabular}{lccccccc}
\hline \multicolumn{1}{c}{ SubCuenca } & \multicolumn{2}{c}{ Centroide } & $\begin{array}{c}\text { Área } \\
\left(\mathrm{Km}^{2}\right)\end{array}$ & $\begin{array}{c}\mathrm{S} \\
(\mathrm{m} / \mathrm{m})\end{array}$ & $\begin{array}{c}\text { Ia } \\
(\mathrm{mm})\end{array}$ & $\begin{array}{c}\text { CN } \\
(\mathrm{SCS})\end{array}$ & $\begin{array}{c}\text { TLag } \\
(\mathrm{min})\end{array}$ \\
\hline Parte Alta & 867378.00 & 1073195.46 & 327.95 & 0.5574 & 51.3 & 49.757 & 189.59 \\
Perrillo & 867204.01 & 1058503.29 & 161.12 & 0.5174 & 41.0 & 55.362 & 169.49 \\
Santo Domingo & 880167.10 & 1069979.67 & 89.15 & 0.4747 & 53.6 & 48.648 & 103.73 \\
Parte Media & 884204.93 & 1063931.89 & 48.33 & 0.5468 & 49.5 & 50.655 & 80.150 \\
San Juan & 887473.93 & 1073632.54 & 61.20 & 0.4675 & 61.5 & 45.247 & 77.859 \\
Barreto & 891295.81 & 1068903.31 & 31.53 & 0.3526 & 47.4 & 51.748 & 83.721 \\
Parte Baja & 902228.87 & 1077564.14 & 67.74 & 0.3822 & 75.8 & 40.116 & 129.43 \\
\hline Fuente- Elaboracín Propla
\end{tabular}

Tabla 3. Parámetros para tramos de cauce.

\begin{tabular}{lcccccc}
\hline Nombre & $\begin{array}{c}\text { Longitud } \\
(\mathrm{m})\end{array}$ & $\begin{array}{c}\mathrm{S} \\
(\mathrm{m} / \mathrm{m})\end{array}$ & Manning & Sección & $\begin{array}{c}\text { Ancho } \\
(\mathrm{m})\end{array}$ & $\mathrm{xH}: 1 \mathrm{~V}$ \\
\hline Guarino2 & 23605.0 & 0.024 & 0.06 & Trapezoidal & 200 & 0.5 \\
Guarino3 & 1417.3 & 0.026 & 0.06 & Trapezoidal & 150 & 0.5 \\
Guarino4 & 1757.8 & 0.025 & 0.06 & Trapezoidal & 150 & 0.3 \\
Guarino5 & 4611.6 & 0.015 & 0.07 & Trapezoidal & 150 & 0.3 \\
Guarino6 & 17383.0 & 0,025 & 0.07 & Trapezoidal & 100 & 0.3 \\
\hline
\end{tabular}

Fuente: Elaboración Propia 
Tabla 4. Análisis de Frecuencia - Estación Marulanda.

\begin{tabular}{cccccc}
\hline $\begin{array}{c}\text { T } \\
\text { Años }\end{array}$ & P & $\begin{array}{c}\text { PP }(\mathrm{mm}) \\
\text { Normal }\end{array}$ & $\begin{array}{c}\text { PP }(\mathrm{mm}) \\
\text { Log-Normal }\end{array}$ & $\begin{array}{c}\text { PP }(\mathrm{mm}) \\
\text { Log-Pearson III }\end{array}$ & $\begin{array}{c}\text { PP }(\mathrm{mm}) \\
\text { Gumbel }\end{array}$ \\
\hline 25 & 0.040 & 78.65 & 80.60 & 85.46 & 83.28 \\
50 & 0.020 & 83.44 & 87.82 & 96.63 & 91.94 \\
100 & 0.010 & 87.75 & 94.87 & 108.47 & 100.54 \\
500 & 0.002 & 96.46 & 110.92 & 138.79 & 120.40 \\
1000 & 0.001 & 99.81 & 117.78 & 153.12 & 128.94 \\
\hline
\end{tabular}

Tabla 5. Análisis de Frecuencia - Estación La Victoria.

\begin{tabular}{cccccc}
\hline $\begin{array}{c}\text { T } \\
\text { años }\end{array}$ & $P$ & $\begin{array}{c}\text { PP }(\mathrm{mm}) \\
\text { Normal }\end{array}$ & $\begin{array}{c}\text { PP }(\mathrm{mm}) \\
\text { Log-Normal }\end{array}$ & $\begin{array}{c}\text { PP }(\mathrm{mm}) \\
\text { Log-Pearson III }\end{array}$ & $\begin{array}{c}\text { PP }(\mathrm{mm}) \\
\text { Gumbel }\end{array}$ \\
\hline 25 & 0.040 & 189.76 & 192.92 & 196.98 & 198.03 \\
100 & 0.020 & 198.32 & 204.50 & 211.40 & 213.51 \\
500 & 0.010 & 206.01 & 215.50 & 225.67 & 228.88 \\
1000 & 0.002 & 221.59 & 239.61 & 258.84 & 264.39 \\
\hline
\end{tabular}

Tabla6. Análisis de Frecuencia - Estación Albania.

\begin{tabular}{cccccc}
\hline $\begin{array}{c}\mathrm{T} \\
\text { años }\end{array}$ & $\mathrm{P}$ & $\begin{array}{c}\text { PP }(\mathrm{mm}) \\
\text { Normal }\end{array}$ & $\begin{array}{c}\text { PP }(\mathrm{mm}) \\
\text { Log-Normal }\end{array}$ & $\begin{array}{c}\text { PP }(\mathrm{mm}) \\
\text { Log-Pearson III }\end{array}$ & $\begin{array}{c}\text { PP }(\mathrm{mm}) \\
\text { Gumbel }\end{array}$ \\
\hline 25 & 0.040 & 241.68 & 229.61 & 271.09 & 256.82 \\
50 & 0.020 & 257.36 & 248.51 & 335.17 & 285.20 \\
100 & 0.010 & 271.46 & 266.83 & 406.61 & 313.36 \\
500 & 0.002 & 300.01 & 308.15 & 558.61 & 378.45 \\
1000 & 0.001 & 310.97 & 325.67 & 592.37 & 406.43 \\
\hline
\end{tabular}

Tabla 7. Análisis de Frecuencia - Estación Cañaveral.

\begin{tabular}{cccccc}
\hline $\mathrm{T}$ & $\mathrm{P}$ & $\mathrm{PP}(\mathrm{mm})$ & $\begin{array}{c}\mathrm{PP}(\mathrm{mm}) \\
\text { Log-Normal }\end{array}$ & $\begin{array}{c}\text { PP }(\mathrm{mm}) \\
\text { Log-Pearson III }\end{array}$ & $\begin{array}{c}\text { PP }(\mathrm{mm}) \\
\text { Gumbel }\end{array}$ \\
\hline años & & 208.42 & 212.01 & 221.29 & 219.21 \\
50 & 0.040 & 219.59 & 227.86 & 244.25 & 239.43 \\
100 & 0.020 & 229.64 & 243.11 & 267.91 & 259.50 \\
500 & 0.010 & 249.98 & 277.19 & 326.21 & 305.87 \\
1000 & 0.002 & 257.79 & 291.52 & 352.89 & 325.81 \\
\hline
\end{tabular}

Fuente: Elaboración Propia

Tabla 8. Análisis de Frecuencia - Estación La Esperanza.

\begin{tabular}{cccccc}
\hline $\begin{array}{c}\mathrm{T} \\
\text { años }\end{array}$ & $\mathrm{P}$ & $\begin{array}{c}\text { PP }(\mathrm{mm}) \\
\text { Normal }\end{array}$ & $\begin{array}{c}\text { PP }(\mathrm{mm}) \\
\text { Log-Normal }\end{array}$ & $\begin{array}{c}\text { PP (mm) } \\
\text { Log-Pearson III }\end{array}$ & $\begin{array}{c}\text { PP }(\mathrm{mm}) \\
\text { Gumbel }\end{array}$ \\
\hline 25 & 0.040 & 65.15 & 69.06 & 69.40 & 76.08 \\
50 & 0.020 & 69.22 & 76.01 & 83.72 & 76.44 \\
100 & 0.010 & 72.88 & 82.85 & 100.36 & 100.65 \\
500 & 0.002 & 80.29 & 98.64 & 107.65 \\
\hline 1000 & 0.001 & 83.14 & 105.48 & 107.92 \\
\hline Fuente: Elaboración Propia & &
\end{tabular}

\section{c. Prueba de bondad de ajuste para las diferentes estaciones.}

Se utilizó el método Kolgomorov - Smimov, para determinar el mejor ajuste entre los valores calculados y valores esperados, la variable son las precipitaciones. El Tabla 9muestra la distribución de frecuencia que mejor se ajusta a cada estación.

En el Tabla 10, muestra las precipitaciones para diferente periodo de retorno según su distribución de frecuencia, después de la prueba de bondad de ajuste

\section{d. Mapas de Isoyetas}

Con las precipitación del Tabla 10, se generaron los mapas de Isoyetas para diferente periodos de retorno, ver Figura 4 , (isoyeta $\mathrm{TR}=25$,) de igual manera para los otros TR

\section{e. Distribución horaria de la Precipitación por Subcuenca}

A partir de los mapas de Isoyetas se obtuvieron las precipitación para cada una de las 7 Subcuenca, y tomando la recomendación U. S. Dept. of Agriculture, Soil Conservation Service, 1973, 1986³ , se elaboraron la distribución horaria de la precipitación para cada Subcuenca, ver Tablas 11, 12, 13, 14, 15, 16 y 17. 
Tabla 9. Distribución de frecuencia ajustada por estaciones.

\begin{tabular}{lc}
\hline \multicolumn{1}{c}{ Estaciones } & Distribución de Frecuencia ajustada \\
\hline Marulanda & Gumbel \\
La Victoria & Log Pearson III \\
Albania & Gumbel \\
Cañaveral & Gumbel \\
La esperanza & Log Normal 2P \\
\hline Fuente: Elaboración Propia &
\end{tabular}

Tabla 10. Precipitación para diferente periodo de retorno.

\begin{tabular}{lccccc}
\hline \multirow{2}{*}{ Estaciones } & \multicolumn{5}{c}{$\mathrm{PP}(\mathrm{mm})$} \\
\cline { 2 - 6 } & $\mathrm{TR}=25$ & $\mathrm{TR}=50$ & $\mathrm{TR}=100$ & $\mathrm{TR}=500$ & $\mathrm{TR}=1000$ \\
\hline Marulanda & 83.28 & 91.94 & 100.54 & 120.40 & 128.94 \\
La Victoria & 196.98 & 211.40 & 225.67 & 258.84 & 273.31 \\
Albania & 256.82 & 285.20 & 313.36 & 378.45 & 406.43 \\
Cañaveral & 219.21 & 239.43 & 259.50 & 305.87 & 325.81 \\
La Esperanza & 69.06 & 76.01 & 82.85 & 98.64 & 105.48 \\
\hline
\end{tabular}

Fuente: Elaboración Propia

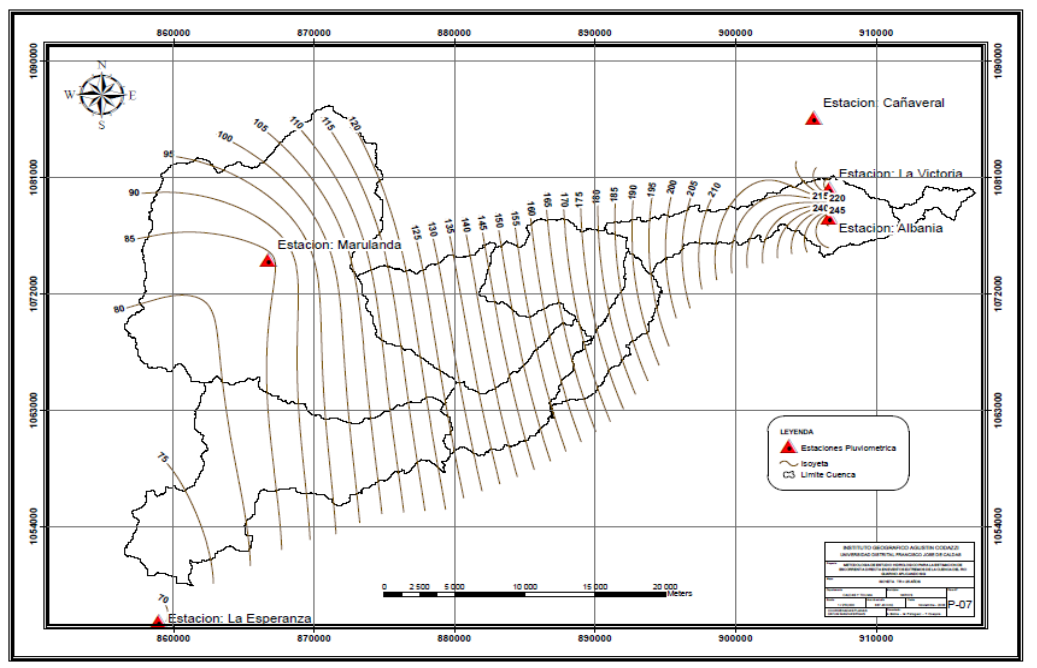

Figura 4. Mapa de Isoyeta $T R=25$ años

Tabla 11. Distribución Horaria de la precipitación Subcuenca Parte Alta.

\begin{tabular}{cccccccc}
\hline T Hora & T HH:Min & Fracción & TR $=25$ & TR $=50$ & TR $=100$ & TR $=500$ & TR $=1000$ \\
\hline 0.0 & $00: 00$ & 0.00 & 0.000 & 0.000 & 0.000 & 0.000 & 0.000 \\
0.6 & $00: 36$ & 0.04 & 3.419 & 3.776 & 4.130 & 5.040 & 5.441 \\
1.2 & $01: 12$ & 0.10 & 8.547 & 9.440 & 10.326 & 12.600 & 13.602 \\
1.5 & $01: 30$ & 0.14 & 11.966 & 13.216 & 14.456 & 17.639 & 19.043 \\
1.8 & $01: 48$ & 0.19 & 16.240 & 17.936 & 19.619 & 23.939 & 25.844 \\
2.1 & $02: 06$ & 0.31 & 26.496 & 29.263 & 32.011 & 39.058 & 42.166 \\
2.3 & $02: 18$ & 0.44 & 37.608 & 41.535 & 45.434 & 55.438 & 59.849 \\
2.4 & $02: 24$ & 0.53 & 45.300 & 50.031 & 54.728 & 66.777 & 72.090 \\
2.5 & $02: 30$ & 0.60 & 51.283 & 56.639 & 61.956 & 75.597 & 81.612 \\
2.6 & $02: 36$ & 0.63 & 53.847 & 59.471 & 65.054 & 79.377 & 85.692 \\
2.8 & $02: 48$ & 0.66 & 56.411 & 62.303 & 68.152 & 83.157 & 89.773 \\
3.0 & $03: 00$ & 0.70 & 59.830 & 66.079 & 72.282 & 88.197 & 95.214 \\
3.3 & $03: 18$ & 0.75 & 64.104 & 70.799 & 77.445 & 94.496 & 102.015 \\
3.6 & $03: 36$ & 0.79 & 67.523 & 74.575 & 81.575 & 99.536 & 107.456 \\
3.9 & $03: 54$ & 0.83 & 70.942 & 78.351 & 85.706 & 104.576 & 112.896 \\
4.2 & $04: 12$ & 0.86 & 73.506 & 81.183 & 88.804 & 108.356 & 116.977 \\
4.5 & $04: 30$ & 0.89 & 76.070 & 84.014 & 91.901 & 112.136 & 121.058 \\
4.8 & $04: 48$ & 0.91 & 77.779 & 85.902 & 93.967 & 114.656 & 123.778 \\
5.4 & $05: 24$ & 0.96 & 82.053 & 90.622 & 99.130 & 120.955 & 130.579 \\
\hline 6.0 & $06: 00$ & 1.00 & 85.472 & 94.398 & 103.260 & 125.995 & 136.020 \\
\hline
\end{tabular}

Fuente: Elaboración Propia 
Tabla 12 - Distribución Horaria de la precipitación Subcuenca Perrillo.

\begin{tabular}{cccccccc}
\hline Hora & T HH:Min & Fracción & TR $=25$ & TR $=50$ & TR $=100$ & TR $=500$ & TR $=1000$ \\
\hline 0.0 & $00: 00$ & 0.00 & 0.000 & 0.000 & 0.000 & 0.000 & 0.000 \\
0.6 & $00: 36$ & 0.04 & 3.406 & 3.770 & 4.129 & 4.965 & 5.341 \\
1.2 & $01: 12$ & 0.10 & 8.515 & 9.424 & 10.323 & 12.414 & 13.352 \\
1.5 & $01: 30$ & 0.14 & 11.921 & 13.194 & 14.453 & 17.379 & 18.692 \\
1.8 & $01: 48$ & 0.19 & 16.179 & 17.906 & 19.615 & 23.586 & 25.368 \\
2.1 & $02: 06$ & 0.31 & 26.397 & 29.215 & 32.003 & 38.482 & 41.390 \\
2.3 & $02: 18$ & 0.44 & 37.466 & 41.466 & 45.423 & 54.619 & 58.748 \\
2.4 & $02: 24$ & 0.53 & 45.130 & 49.947 & 54.714 & 65.792 & 70.764 \\
2.5 & $02: 30$ & 0.60 & 51.090 & 56.544 & 61.941 & 74.481 & 80.110 \\
2.6 & $02: 36$ & 0.63 & 53.645 & 59.372 & 65.038 & 78.205 & 84.116 \\
2.8 & $02: 48$ & 0.66 & 56.199 & 62.199 & 68.135 & 81.929 & 88.121 \\
3.0 & $03: 00$ & 0.70 & 59.605 & 65.968 & 72.264 & 86.895 & 93.462 \\
3.3 & $03: 18$ & 0.75 & 63.863 & 70.680 & 77.426 & 93.101 & 100.138 \\
3.6 & $03: 36$ & 0.79 & 67.269 & 74.450 & 81.555 & 98.067 & 105.479 \\
3.9 & $03: 54$ & 0.83 & 70.675 & 78.220 & 85.685 & 103.032 & 110.819 \\
4.2 & $04: 12$ & 0.86 & 73.229 & 81.047 & 88.782 & 106.756 & 114.825 \\
4.5 & $04: 30$ & 0.89 & 75.784 & 83.874 & 91.879 & 110.480 & 118.830 \\
4.8 & $04: 48$ & 0.91 & 77.487 & 85.759 & 93.944 & 112.963 \\
5.4 & $05: 24$ & 0.96 & 81.744 & 90.471 & 99.105 & 119.170 & 121.501 \\
6.0 & $06: 00$ & 1.00 & 85.150 & 94.241 & 103.235 & 124.135 & 133.177 \\
Fuente: Elaboracion Propia & & & & & & &
\end{tabular}

Tabla 13 - Distribución Horaria de la precipitación Subcuenca Santo Domingo.

\begin{tabular}{cccccccc}
\hline Hora & T HH:Min & Fracción & TR $=25$ & $\mathrm{TR}=50$ & $\mathrm{TR}=100$ & $\mathrm{TR}=500$ & $\mathrm{TR}=1000$ \\
\hline 0.0 & $00: 00$ & 0.00 & 0.000 & 0.000 & 0.000 & 0.000 & 0.000 \\
0.6 & $00: 36$ & 0.04 & 5.301 & 5.865 & 6.424 & 7.512 & 8.003 \\
1.2 & $01: 12$ & 0.10 & 13.252 & 14.661 & 16.059 & 18.781 & 20.007 \\
1.5 & $01: 30$ & 0.14 & 18.553 & 20.526 & 22.483 & 26.293 & 28.010 \\
1.8 & $01: 48$ & 0.19 & 25.179 & 27.856 & 30.512 & 35.684 & 38.013 \\
2.1 & $02: 06$ & 0.31 & 41.082 & 45.450 & 49.783 & 58.221 & 62.021 \\
2.3 & $02: 18$ & 0.44 & 58.310 & 64.510 & 70.660 & 82.636 & 88.030 \\
2.4 & $02: 24$ & 0.53 & 70.237 & 77.705 & 85.114 & 99.539 & 106.036 \\
2.5 & $02: 30$ & 0.60 & 79.514 & 87.968 & 96.355 & 112.686 & 120.041 \\
2.6 & $02: 36$ & 0.63 & 83.489 & 92.366 & 101.173 & 118.320 & 126.043 \\
2.8 & $02: 48$ & 0.66 & 87.465 & 96.764 & 105.991 & 123.954 & 132.045 \\
3.0 & $03: 00$ & 0.70 & 92.766 & 102.629 & 112.414 & 131.467 & 140.048 \\
3.3 & $03: 18$ & 0.75 & 99.392 & 109.959 & 120.444 & 140.857 & 150.051 \\
3.6 & $03: 36$ & 0.79 & 104.693 & 115.824 & 126.868 & 148.370 & 158.054 \\
3.9 & $03: 54$ & 0.83 & 109.994 & 121.688 & 133.291 & 155.882 & 166.057 \\
4.2 & $04: 12$ & 0.86 & 113.970 & 126.087 & 138.109 & 161.516 & 172.059 \\
4.5 & $04: 30$ & 0.89 & 117.945 & 130.485 & 142.927 & 167.151 & 178.061 \\
4.8 & $04: 48$ & 0.91 & 120.596 & 133.417 & 146.139 & 170.907 & 182.062 \\
5.4 & $05: 24$ & 0.96 & 127.222 & 140.748 & 154.168 & 180.297 & 192.066 \\
6.0 & $06: 00$ & 1.00 & 132.523 & 146.613 & 160.592 & 187.810 & 200.068 \\
\hline
\end{tabular}

Tabla 14 - Distribución Horaria de la precipitación Subcuenca Media.

\begin{tabular}{|c|c|c|c|c|c|c|c|}
\hline Hora & T HH:Min & Fracción & $\mathrm{TR}=25$ & $\mathrm{TR}=50$ & $\mathrm{TR}=100$ & $\mathrm{TR}=500$ & $\mathrm{TR}=1000$ \\
\hline 0.0 & $00: 00$ & 0.00 & 0.000 & 0.000 & 0.000 & 0.000 & 0.000 \\
\hline 0.6 & $00: 36$ & 0.04 & 5.790 & 6.420 & 7.046 & 8.147 & 8.649 \\
\hline 1.2 & 01:12 & 0.10 & 14.474 & 16.051 & 17.614 & 20.367 & 21.622 \\
\hline 1.5 & 01:30 & 0.14 & 20.264 & 22.471 & 24.660 & 28.514 & 30.271 \\
\hline 1.8 & 01:48 & 0.19 & 27.501 & 30.496 & 33.467 & 38.697 & 41.083 \\
\hline 2.1 & 02:06 & 0.31 & 44.869 & 49.757 & 54.604 & 63.138 & 67.030 \\
\hline 2.3 & $02: 18$ & 0.44 & 63.686 & 70.623 & 77.503 & 89.615 & 95.139 \\
\hline 2.4 & $02: 24$ & 0.53 & 76.712 & 85.069 & 93.356 & 107.945 & 114.599 \\
\hline 2.5 & $02: 30$ & 0.60 & 86.844 & 96.304 & 105.686 & 122.202 & 129.735 \\
\hline 2.6 & $02: 36$ & 0.63 & 91.186 & 101.119 & 110.970 & 128.312 & 136.221 \\
\hline 2.8 & $02: 48$ & 0.66 & 95.529 & 105.934 & 116.254 & 134.422 & 142.708 \\
\hline 3.0 & 03:00 & 0.70 & 101.318 & 112.355 & 123.300 & 142.569 & 151.357 \\
\hline 3.3 & $03: 18$ & 0.75 & 108.555 & 120.380 & 132.107 & 152.753 & 162.168 \\
\hline 3.6 & $03: 36$ & 0.79 & 114.345 & 126.800 & 139.153 & 160.900 & 170.817 \\
\hline 3.9 & $03: 54$ & 0.83 & 120.134 & 133.221 & 146.199 & 169.046 & 179.466 \\
\hline 4.2 & $04: 12$ & 0.86 & 124.477 & 138.036 & 151.483 & 175.157 & 185.953 \\
\hline 4.5 & $04: 30$ & 0.89 & 128.819 & 142.851 & 156.767 & 181.267 & 192.440 \\
\hline 4.8 & 04:48 & 0.91 & 131.714 & 146.061 & 160.290 & 185.340 & 196.764 \\
\hline 5.4 & $05: 24$ & 0.96 & 138.951 & 154.086 & 169.097 & 195.524 & 207.576 \\
\hline 6.0 & 06:00 & 1.00 & 144.740 & 160.507 & 176.143 & 203.670 & 216.225 \\
\hline
\end{tabular}


Tabla 15 - Distribución Horaria de la precipitación Subcuenca San Juan.

\begin{tabular}{|c|c|c|c|c|c|c|c|}
\hline Hora & T HH:Min & Fracción & $\mathrm{TR}=25$ & $\mathrm{TR}=50$ & $\mathrm{TR}=100$ & $\mathrm{TR}=500$ & $\mathrm{TR}=1000$ \\
\hline 0.0 & $00: 00$ & 0.00 & 0.000 & 0.000 & 0.000 & 0.000 & 0.000 \\
\hline 0.6 & $00: 36$ & 0.04 & 6.671 & 7.371 & 8.066 & 9.331 & 9.902 \\
\hline 1.2 & 01:12 & 0.10 & 16.678 & 18.428 & 20.166 & 23.328 & 24.754 \\
\hline 1.5 & 01:30 & 0.14 & 23.349 & 25.800 & 28.232 & 32.659 & 34.656 \\
\hline 1.8 & $01: 48$ & 0.19 & 31.688 & 35.014 & 38.315 & 44.324 & 47.033 \\
\hline 2.1 & 02:06 & 0.31 & 51.701 & 57.128 & 62.513 & 72.317 & 76.738 \\
\hline 2.3 & $02: 18$ & 0.44 & 73.382 & 81.085 & 88.728 & 102.644 & 108.918 \\
\hline 2.4 & $02: 24$ & 0.53 & 88.393 & 97.671 & 106.877 & 123.639 & 131.197 \\
\hline 2.5 & $02: 30$ & 0.60 & 100.067 & 110.570 & 120.993 & 139.969 & 148.525 \\
\hline 2.6 & $02: 36$ & 0.63 & 105.070 & 116.099 & 127.043 & 146.968 & 155.951 \\
\hline 2.8 & $02: 48$ & 0.66 & 110.074 & 121.627 & 133.092 & 153.966 & 163.377 \\
\hline 3.0 & 03:00 & 0.70 & 116.745 & 128.999 & 141.159 & 163.297 & 173.279 \\
\hline 3.3 & $03: 18$ & 0.75 & 125.084 & 138.213 & 151.241 & 174.961 & 185.656 \\
\hline 3.6 & 03:36 & 0.79 & 131.755 & 145.584 & 159.308 & 184.293 & 195.558 \\
\hline 3.9 & $03: 54$ & 0.83 & 138.426 & 152.956 & 167.374 & 193.624 & 205.459 \\
\hline 4.2 & $04: 12$ & 0.86 & 143.429 & 158.484 & 173.424 & 200.622 & 212.886 \\
\hline 4.5 & 04:30 & 0.89 & 148.433 & 164.013 & 179.473 & 207.621 & 220.312 \\
\hline 4.8 & $04: 48$ & 0.91 & 151.768 & 167.698 & 183.506 & 212.287 & 225.263 \\
\hline 5.4 & $05: 24$ & 0.96 & 160.107 & 176.913 & 193.589 & 223.951 & 237.640 \\
\hline 6.0 & 06:00 & 1.00 & 166.778 & 184.284 & 201.655 & 233.282 & 247.541 \\
\hline
\end{tabular}

Tabla 16 - Distribución Horaria de la precipitación Subcuenca Barreto.

\begin{tabular}{cccccccc}
\hline Hora & T H:Min & Fracción & $\mathrm{TR}=25$ & $\mathrm{TR}=50$ & $\mathrm{TR}=100$ & $\mathrm{TR}=500$ & $\mathrm{TR}=1000$ \\
\hline 0.0 & $00: 00$ & 0.00 & 0.000 & 0.000 & 0.000 & 0.000 & 0.000 \\
0.6 & $00: 36$ & 0.04 & 7.188 & 7.964 & 8.735 & 10.010 & 10.592 \\
1.2 & $01: 12$ & 0.10 & 17.969 & 19.910 & 21.837 & 25.024 & 26.479 \\
1.5 & $01: 30$ & 0.14 & 25.156 & 27.874 & 30.571 & 35.034 & 37.071 \\
1.8 & $01: 48$ & 0.19 & 34.141 & 37.830 & 41.489 & 47.546 & 50.310 \\
2.1 & $02: 06$ & 0.31 & 55.703 & 61.722 & 67.693 & 77.574 & 82.085 \\
2.3 & $02: 18$ & 0.44 & 79.063 & 87.605 & 96.081 & 110.106 & 116.508 \\
2.4 & $02: 24$ & 0.53 & 95.235 & 105.525 & 115.734 & 132.627 & 140.339 \\
2.5 & $02: 30$ & 0.60 & 107.813 & 119.462 & 131.019 & 150.144 & 158.874 \\
2.6 & $02: 36$ & 0.63 & 113.204 & 125.435 & 137.570 & 157.651 & 166.818 \\
2.8 & $02: 48$ & 0.66 & 118.594 & 131.408 & 144.121 & 165.159 & 174.761 \\
3.0 & $03: 00$ & 0.70 & 125.782 & 139.372 & 152.856 & 175.168 & 185.353 \\
3.3 & $03: 18$ & 0.75 & 134.766 & 149.327 & 163.774 & 187.680 & 198.593 \\
3.6 & $03: 36$ & 0.79 & 141.954 & 157.292 & 172.509 & 197.690 & 209.184 \\
3.9 & $03: 54$ & 0.83 & 149.141 & 165.256 & 181.243 & 207.699 & 219.776 \\
4.2 & $04: 12$ & 0.86 & 154.532 & 171.229 & 187.794 & 215.207 & 227.719 \\
4.5 & $04: 30$ & 0.89 & 159.923 & 177.202 & 194.345 & 222.714 & 235.663 \\
4.8 & $04: 48$ & 0.91 & 163.516 & 181.184 & 198.712 & 227.719 & 240.959 \\
5.4 & $05: 24$ & 0.96 & 172.501 & 191.139 & 209.631 & 240.231 & 254.198 \\
6.0 & $06: 00$ & 1.00 & 179.688 & 199.103 & 218.365 & 250.240 & 264.790 \\
\hline Fuente: Elaboracion Propia & & & & & &
\end{tabular}

Tabla 17 - Distribución Horaria de la precipitación Subcuenca Parte Baja.

\begin{tabular}{cccccccc}
\hline Hora & T HH:Min & Fracción & $\mathrm{TR}=25$ & $\mathrm{TR}=50$ & $\mathrm{TR}=100$ & $\mathrm{TR}=500$ & $\mathrm{TR}=1000$ \\
\hline 0.0 & $00: 00$ & 0.00 & 0.000 & 0.000 & 0.000 & 0.000 & 0.000 \\
0.6 & $00: 36$ & 0.04 & 9.088 & 10.017 & 10.939 & 12.602 & 13.337 \\
1.2 & $01: 12$ & 0.10 & 22.721 & 25.043 & 27.348 & 31.504 & 33.344 \\
1.5 & $01: 30$ & 0.14 & 31.809 & 35.061 & 38.287 & 44.105 & 46.681 \\
1.8 & $01: 48$ & 0.19 & 43.170 & 47.583 & 51.960 & 59.857 & 63.353 \\
2.1 & $02: 06$ & 0.31 & 70.435 & 77.635 & 84.777 & 97.662 & 103.365 \\
2.3 & $02: 18$ & 0.44 & 99.972 & 110.191 & 120.329 & 138.617 & 146.712 \\
2.4 & $02: 24$ & 0.53 & 120.421 & 132.730 & 144.942 & 166.970 & 176.721 \\
2.5 & $02: 30$ & 0.60 & 136.326 & 150.261 & 164.085 & 189.023 & 200.062 \\
2.6 & $02: 36$ & 0.63 & 143.142 & 157.774 & 172.289 & 198.474 & 210.065 \\
2.8 & $02: 48$ & 0.66 & 149.958 & 165.287 & 180.494 & 207.925 & 220.068 \\
3.0 & $03: 00$ & 0.70 & 159.046 & 175.304 & 191.433 & 220.527 & 233.405 \\
3.3 & $03: 18$ & 0.75 & 170.407 & 187.826 & 205.106 & 236.278 & 250.077 \\
3.6 & $03: 36$ & 0.79 & 179.495 & 197.843 & 216.045 & 248.880 & 263.415 \\
3.9 & $03: 54$ & 0.83 & 188.584 & 207.861 & 226.984 & 261.481 & 276.752 \\
4.2 & $04: 12$ & 0.86 & 195.400 & 215.374 & 235.189 & 270.933 & 286.755 \\
4.5 & $04: 30$ & 0.89 & 202.216 & 222.887 & 243.393 & 280.384 & 296.758 \\
4.8 & $04: 48$ & 0.91 & 206.760 & 227.895 & 248.862 & 286.684 & 303.427 \\
5.4 & $05: 24$ & 0.96 & 218.121 & 240.417 & 262.536 & 302.436 & 320.099 \\
6.0 & $06: 00$ & 1.00 & 227.209 & 250.435 & 273.475 & 315.038 & 333.436 \\
\hline Fuente: Elaboracion Propia & & & & & & &
\end{tabular}




\section{f. Generación de Descarga}

Los caudales máximos obtenidos para los diferentes periodos de retornos se muestran en las Tablas 18, 19, 20, 21 y 22, se observan precipitaciones totales para cada Subcuenca, y las pérdidas totales calculada por el programa varían según los parámetros utilizados en el modelo de la cuenca, el exceso de precipitación es la lluvia efectiva (es la diferencia entre la precipitación total y las pérdida total), que es transformado en descarga total por cada Subcuenca. El caudal máximo es proporcional al tamaño de cada Subcuenca, de modo que los caudales específicos estimados muestran que para una determinada condición de la cuenca, $1 \mathrm{~km}^{2}$ genera cierta cantidad de caudal, de manera que las Subcuenca que tienen caudal especifico menor son las que tienen mayor pérdida al generar el escurrimiento.

La escorrentía varía en función del tiempo, en estas tablas, lo que se muestra es el caudal pico (caudal máximo), para diferente periodo de retorno.

Tabla 18 - Resultados generados por el HEC HMS por SubcuencaTR $=25$ años.

\begin{tabular}{cccccccc}
\hline Subcuenca & $\begin{array}{c}\text { Precip. total } \\
(\mathrm{mm})\end{array}$ & $\begin{array}{c}\text { Pérdida total } \\
(\mathrm{mm})\end{array}$ & $\begin{array}{c}\text { Exceso total } \\
(\mathrm{mm})\end{array}$ & $\begin{array}{c}\text { Descarga total } \\
(\mathrm{mm})\end{array}$ & $\begin{array}{c}\text { Área } \\
\left(\mathrm{km}^{2}\right)\end{array}$ & $\begin{array}{c}\text { Caudal Máximo } \\
\left(\mathrm{m}^{3} / \mathrm{s}\right)\end{array}$ & $\begin{array}{c}\text { Caudal Especif. } \\
\left(\mathrm{m}^{3} / \mathrm{s} \mathrm{km}^{2} /\right)\end{array}$ \\
\hline Parte Alta & 85.47 & 81.45 & 4.02 & 4.02 & 327.95 & 66.3 & 0.20 \\
Perrillo & 85.15 & 77.32 & 7.83 & 7.83 & 161.12 & 65.3 & 0.41 \\
Santo Domingo & 132.52 & 114.57 & 17.95 & 17.95 & 89.15 & 97.4 & 1.09 \\
Parte Media & 144.74 & 118.27 & 26.47 & 26.47 & 48.33 & 82.1 & 1.70 \\
San Juan & 166.78 & 139.92 & 26.86 & 26.86 & 61.20 & 105.9 & 1.73 \\
Barreto & 179.69 & 132.28 & 47.41 & 47.41 & 31.53 & 96.4 & 3.06 \\
Parte Baja & 227.21 & 184.00 & 43.21 & 43.21 & 67.74 & 165.7 & 2.45 \\
\hline
\end{tabular}

Fuente: Elaboración Propia

Tabla 19. Resultados generados por el HEC HMS por Subcuenca TR $=50$ años.

\begin{tabular}{|c|c|c|c|c|c|c|c|}
\hline Subcuenca & $\begin{array}{l}\text { Precip. total } \\
\text { (mm) }\end{array}$ & $\begin{array}{l}\text { Pérdida total } \\
(\mathrm{mm})\end{array}$ & $\begin{array}{l}\text { Exceso total } \\
(\mathrm{mm})\end{array}$ & $\begin{array}{l}\text { Descarga total } \\
(\mathrm{mm})\end{array}$ & $\begin{array}{l}\text { Área } \\
\left(\mathrm{km}^{2}\right)\end{array}$ & $\begin{array}{l}\text { Caudal Máximo } \\
\left(\mathrm{m}^{3} / \mathrm{s}\right)\end{array}$ & $\begin{array}{l}\text { Caudal Especif. } \\
\left(\mathrm{m}^{3} / \mathrm{s} \mathrm{km}^{2} /\right)\end{array}$ \\
\hline Parte Alta & 94.40 & 88.20 & 6.20 & 6.20 & 327.95 & 99.1 & 0.30 \\
\hline Perrillo & 94.24 & 83.26 & 10.99 & 10.99 & 161.12 & 90.7 & 0.56 \\
\hline Santo Domingo & 146.61 & 122.65 & 23.96 & 23.96 & 89.15 & 127.6 & 1.43 \\
\hline Parte Media & 160.51 & 126.13 & 34.38 & 34.38 & 48.33 & 1069 & $\begin{array}{l}1.47 \\
2.21\end{array}$ \\
\hline San Juan & 184.28 & 149.23 & 35.05 & 35.05 & 61.20 & 138.3 & 2.26 \\
\hline Barreto & 199.10 & 139.87 & 59.23 & 59.23 & 31.53 & 120.6 & 3.82 \\
\hline Parte Baja & 250.44 & 195.37 & 55.07 & 55.07 & 67.74 & 211.1 & 3.12 \\
\hline
\end{tabular}

Tabla 20 - Resultados generados por el HEC HMS por Subcuenca TR = 100 años .

\begin{tabular}{cccccccc}
\hline Subcuenca & $\begin{array}{c}\text { Precip. Total } \\
(\mathrm{mm})\end{array}$ & $\begin{array}{c}\text { Pérdida total } \\
(\mathrm{mm})\end{array}$ & $\begin{array}{c}\text { Exceso Total } \\
(\mathrm{mm})\end{array}$ & $\begin{array}{c}\text { Descarga total } \\
(\mathrm{mm})\end{array}$ & $\begin{array}{c}\text { Área } \\
\left(\mathrm{km}^{2}\right)\end{array}$ & $\begin{array}{c}\text { Caudal Máximo Caudal Especif. } \\
\left(\mathrm{m}^{3} / \mathrm{s}\right)\end{array}$ & $\begin{array}{c}\text { E } \\
\left(\mathrm{m}^{3} / \mathrm{s} \mathrm{km}^{2}\right)\end{array}$ \\
\hline Parte Alta & 103.26 & 94.51 & 8.75 & 8.75 & 327.95 & 136.3 & 0.42 \\
Perrillo & 103.24 & 88.73 & 14.51 & 14.51 & 161.12 & 118.9 & 0.74 \\
Santo Domingo & 160.59 & 130.07 & 30.52 & 30.52 & 89.15 & 161.3 & 1.81 \\
Parte Media & 176.14 & 133.27 & 42.87 & 42.87 & 48.33 & 133.5 & 2.76 \\
San Juan & 201.66 & 157.76 & 43.90 & 43.90 & 61.20 & 173.3 & 2.83 \\
Barreto & 218.36 & 146.69 & 71.67 & 71.67 & 31.53 & 145.9 & 4.63 \\
Parte Baja & 273.48 & 205.74 & 67.74 & 67.74 & 67.74 & 259.4 & 3.83 \\
\hline
\end{tabular}

Fuente: Elaboración Propia

Tabla 21 - Resultados generados por el HEC HMS por Subcuenca TR = 500 años.

\begin{tabular}{lccccccc}
\hline \multicolumn{1}{c}{ Subcuenca } & $\begin{array}{c}\text { Precip. total } \\
(\mathrm{mm})\end{array}$ & $\begin{array}{c}\text { Pérdida total } \\
(\mathrm{mm})\end{array}$ & $\begin{array}{c}\text { Exceso total } \\
(\mathrm{mm})\end{array}$ & $\begin{array}{c}\text { Descarga Total } \\
(\mathrm{mm})\end{array}$ & $\begin{array}{c}\text { Área } \\
\left(\mathrm{km}^{2}\right)\end{array}$ & $\begin{array}{c}\text { Caudal Máximo Caudal Especif. } \\
\left(\mathrm{m}^{3} / \mathrm{s}\right)\end{array}$ & $\begin{array}{c}\text { Can } \\
\left(\mathrm{m}^{3} / \mathrm{s} \mathrm{km}^{2} /\right)\end{array}$ \\
\hline Parte Alta & 126.00 & 109.15 & 16.85 & 16.85 & 327.95 & 263.1 & 0.80 \\
Perrillo & 124.14 & 100.13 & 24.01 & 24.01 & 161.12 & 194.1 & 1.20 \\
Santo Domingo & 187.81 & 143.04 & 44.77 & 44.77 & 89.15 & 243.1 & 2.73 \\
Parte Media & 203.67 & 144.49 & 59.18 & 59.18 & 48.33 & 184.4 & 3.82 \\
San Juan & 233.38 & 171.69 & 61.59 & 61.59 & 61.20 & 242.9 & 3.97 \\
Barreto & 250.24 & 156.66 & 93.58 & 93.58 & 31.53 & 192.3 & 6.10 \\
Parte Baja & 315.04 & 222.49 & 92.55 & 92.55 & 67.74 & 353.6 & 5.22 \\
\hline
\end{tabular}

Fuente: Elaboración Propia 
Tabla 22 - Resultados generados por el HEC HMS por Subcuenca TR = 1000 años.

\begin{tabular}{|c|c|c|c|c|c|c|c|}
\hline Subcuenca & $\begin{array}{l}\text { Precip. total } \\
\text { (mm) }\end{array}$ & $\begin{array}{l}\text { Pérdida total } \\
(\mathrm{mm})\end{array}$ & $\begin{array}{l}\text { Exceso total } \\
(\mathrm{mm})\end{array}$ & $\begin{array}{l}\text { Descarga total } \\
(\mathrm{mm})\end{array}$ & $\begin{array}{l}\text { Área } \\
\left(\mathrm{km}^{2}\right)\end{array}$ & $\begin{array}{c}\text { Caudal Máximo } \\
\left(\mathrm{m}^{3} / \mathrm{s}\right)\end{array}$ & $\begin{array}{c}\text { Caudal Especif. } \\
\left(\mathrm{m}^{3} / \mathrm{s} \mathrm{km}^{2} /\right)\end{array}$ \\
\hline$\overline{\text { Parte Alta }}$ & 136.02 & 114.98 & 21.04 & 21.04 & 327.95 & 328.6 & 1.00 \\
\hline Perrillo & 133.52 & 104.73 & 28.79 & 28.79 & 161.12 & 231.6 & 1.44 \\
\hline Santo Domingo & 200.07 & 148.32 & 51.75 & 51.75 & 89.15 & 283.6 & 3.18 \\
\hline Parte Media & 216.22 & 149.11 & 67.11 & 67.11 & 48.33 & 211.5 & 4.38 \\
\hline San Juan & 247.54 & 177.39 & 70.15 & 70.15 & 61.20 & 280.7 & 4.59 \\
\hline Barreto & 264.79 & 160.75 & 104.04 & 104.04 & 31.53 & 216.0 & 6.85 \\
\hline Parte Baja & 333.44 & 229.20 & 104.24 & 104.24 & 67.74 & 397.5 & 5.87 \\
\hline
\end{tabular}

Fuente: Elaboración Propia

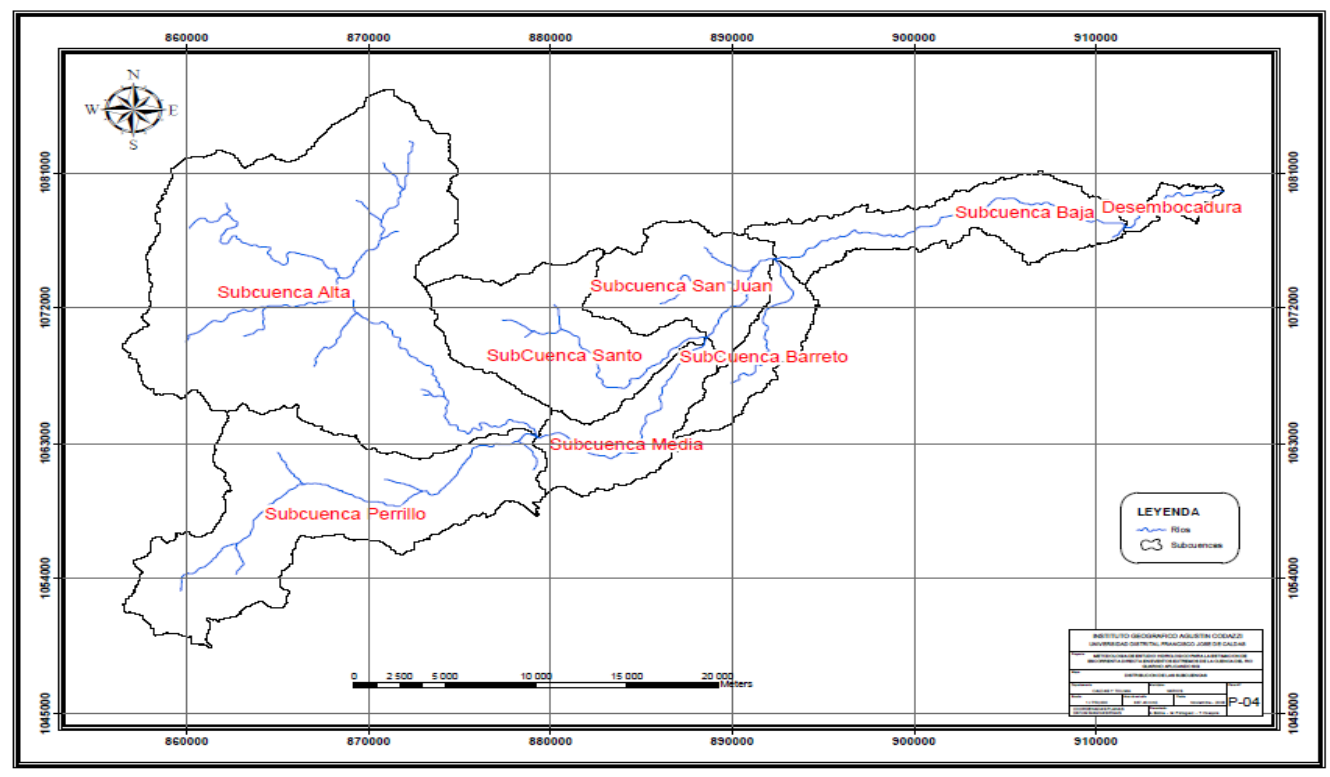

Figura 5. Cuenca Rio Guarino - División en Subcuencas

Tabla 23 - Descargas Máximas - Cuenca Guarinó.

\begin{tabular}{lccccc}
\hline \multirow{2}{*}{ Subcuenca } & \multicolumn{5}{c}{ Descarga máxima directa $\left(\mathrm{m}^{3} / \mathrm{s}\right)$} \\
\cline { 2 - 5 } & $\mathrm{TR}=25$ & $\mathrm{TR}=50$ & $\mathrm{TR}=100$ & $\mathrm{TR}=500$ & $\mathrm{TR}=1000$ \\
\hline Parte Alta & 66.3 & 99.1 & 136.3 & 263.1 & 328.6 \\
Perrillo & 65.3 & 90.7 & 118.9 & 194.1 & 231.6 \\
Santo Domingo & 97.4 & 127.6 & 161.3 & 243.1 & 283.6 \\
Parte Media & 82.1 & 106.9 & 133.5 & 184.4 & 211.5 \\
San Juan & 105.9 & 138.3 & 173.3 & 242.9 & 280.7 \\
Barreto & 96.4 & 120.6 & 145.9 & 192.3 & 216.0 \\
Parte Baja & 165.7 & 211.1 & 259.4 & 353.6 & 397.5 \\
\hline \multicolumn{1}{c}{ Total } & 445.3 & 578.4 & 783.3 & 1128.8 & 1328.3 \\
\hline Fuente: Elaboración Propia & \multicolumn{5}{c}{}
\end{tabular}


El Tabla 23, muestra descarga directa para cada periodo de retorno por subcuenca. (Ver figura 5, división en Subcuencas)

Las figuras 6, 7, 8, 9 y 10 se observa los hidrogramas de salida de la Cuenca del río Guarinó, para diferentes periodos de retornos.

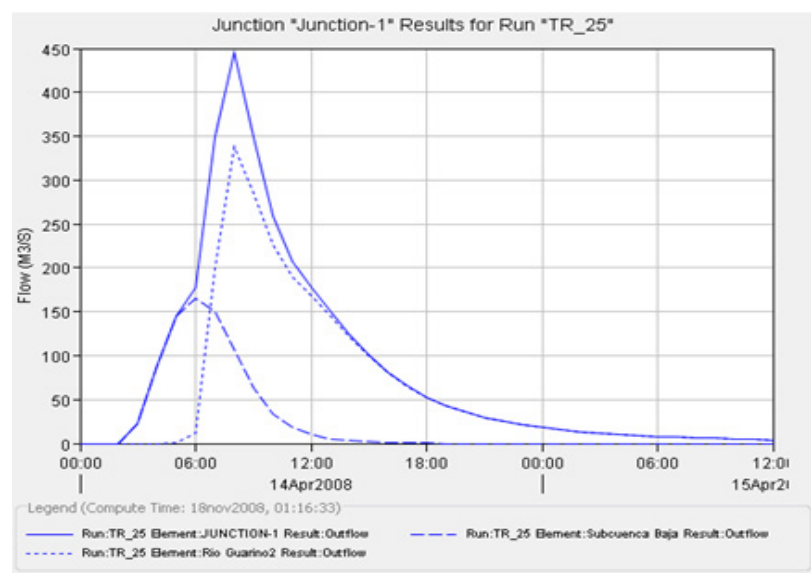

Figura 6. Hidrográma de salida $\mathrm{TR}=25$ años.

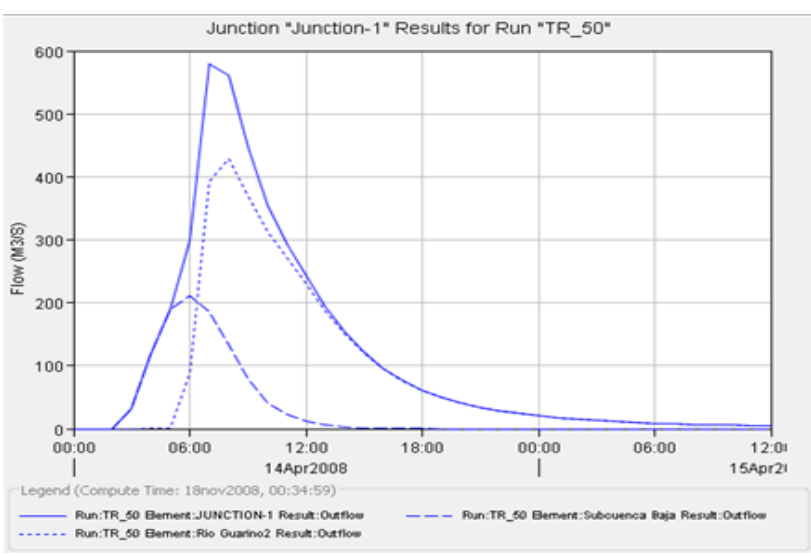

Figura 7. Hidrograma de salida $\mathrm{TR}=50$ años.

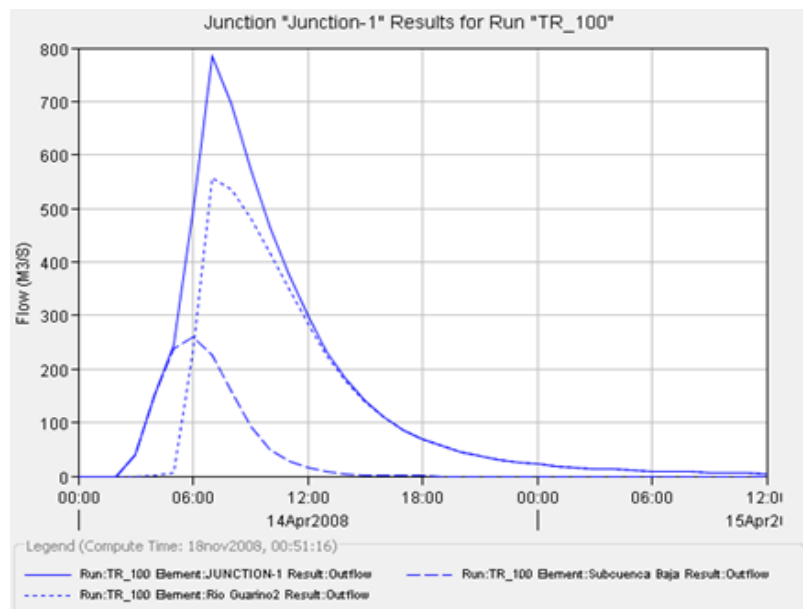

Figura 8. Hidrográma de salida TR $=100$ años.

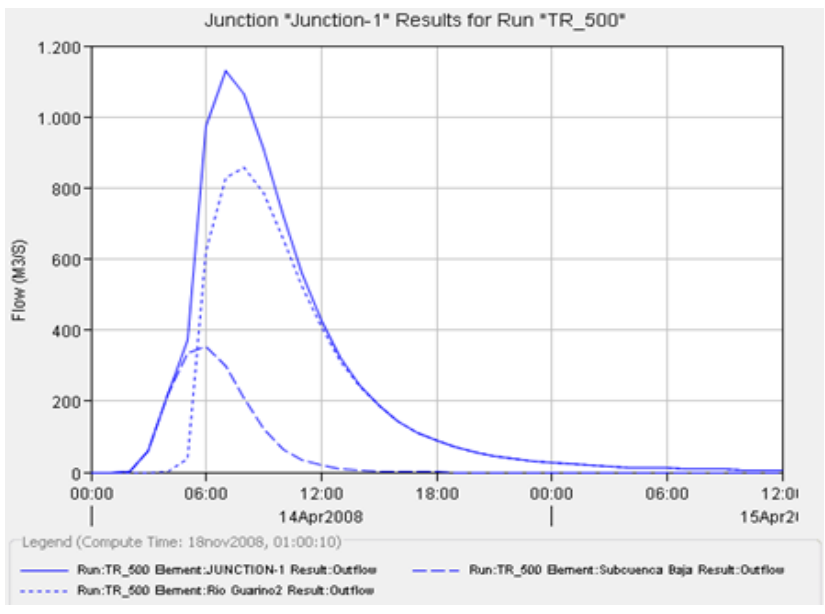

Figura 9. Hidrograma de salida TR $=500$ años.

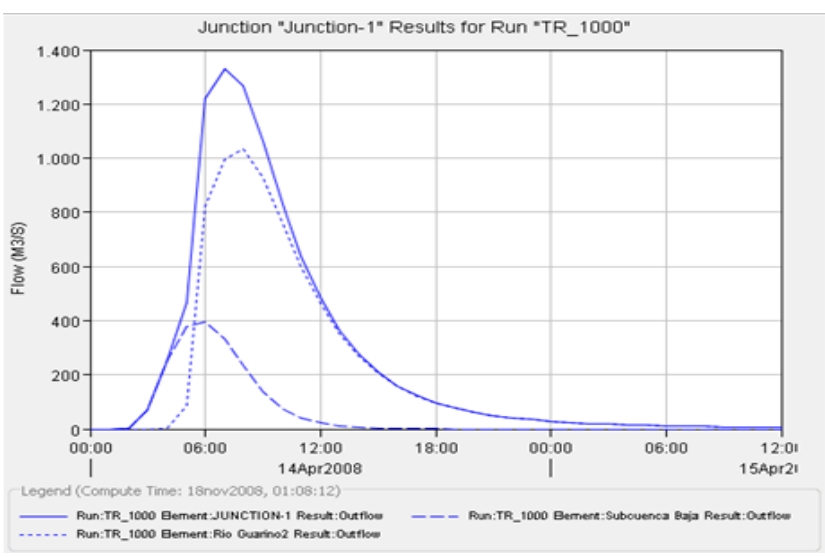

Figura 14. Hidrográma de salida TR $=1000$ años.

\section{Conclusiones}

Ante métodos racionales o modelos empíricos, la metodología planteada es una buena alternativa de estimar la escorrentía, esto debido a la espacialización de las variables que intervienen en el ciclo hidrológico.

La aplicación del programa de modelamiento hidrológico HEC HMS, se están utilizando con resultados aceptables, esta metodología incorpora los sistemas de información geográfica que nos permite obtener las características hidrológicas de cada Subcuenca o microcuencas, que conforman el área en estudio.

Con información de los mapas de cobertura y uso, tipo de suelo y DEM, fue posible generar un mapa de Curva Numero de la cuenca, que nos permitió cuantificar tanto las perdida y el exceso de precipitación.

Con información de las precipitaciones máximas en 24 horas de las 5 estaciones, aplicando las distribuciones de frecuencia de las precipitaciones se generaron las Isoyetas para periodos de retornos de 25, 50, 100, 500 y 1000 años, este mapa nos permitió cuantificar las precipitaciones máximas totales que fueron utilizados en el modelo.

La escorrentía directa estimada en el punto de control de la cuenca del río Guarinó, para los periodos de retorno de 
25, 50, 100, 500 y 1000 años, es de 445.3, 578.4, 783.3, 1128.8 y $1328.3 \mathrm{~m}^{3} / \mathrm{s}$ respectivamente.

Conocer la escorrentía directa en evento extremo es de suma importancia, como datos de diseños en aliviaderos, sistema de drenaje en carreteras, aeropuertos, ciudades, entre otros.

\section{Recomendaciones}

Las cuencas hidrográficas son sistemas abiertos de procesos y respuestas, en cuyo comportamiento participan factores físicos y humanos, son altamente dinámicas, por lo cual es importante actualizar los mapa de cobertura y uso de suelos, variable altamente vulnerable.

Un aspecto que se debe tomar en cuenta es que no existe estaciones automatizadas en la zona donde los parámetros hidrológicos sean medidos y registrados por lo tanto se corre el riesgo de precisar los resultados.

Es recomendable contar con mayor número de estaciones, que estén ubicadas estratégicamente, ya que es una variable de suma importancia en el modelo

Se recomienda contar con registros de caudales máximos anuales en el punto de control y Flujo base, para poder realizar una calibración en el modelo, con los datos medidos y estimados, descontando el caudal base.

\section{Literatura citada}

Camarasa, A.; Blasco, A. y Lopez, M. 2001. Análisis mediante SIG de los parámetros de producción de escorrentía, centro de investigaciones sobre desertificación, departamento de geografía. Universidad de Valencia - España.

Castillo, E. G.; Corredor, J. L. y Castillo, S. P. 2007. Evaluación de la oferta hídrica superficial usando herramientas SIG, Revista Análisis Geográficos No 37 , IGAC, Bogotá.

Chavarri, E. 2008. Métodos de Análisis En Recurso de Agua, Notas de clase, Universidad Agraria La Molina, Maestría en Recursos Hídricos - Lima.

Chow, T. y Maidment, D. R. 1994. Hidrología Aplicada, Editorial Mc GRAW HILL - Primera Edición, Bogotá Colombia.

Martinez, J. C. 1996. Modelo lluvia - Escorrentía basado en el método del Soil Conservation Service para cuencas Colombianas, Memoria de Investigación N 302 Universidad de los Andes Bogotá Octubre.

Montserrat, F. y Rodriguez, J. y Estrela, T. 1995. Generación Automática del Número e Curva con Sistemas de Información Geográfica, Revista Ingeniería del Agua. Vol 2 Número 4, Valencia, Diciembre.

IGAC, 2007, Mapa de Red Hídrica de la Cuenca del Río Guarinó Mapa 1:100000 Bogotá.

IGAC, 2007, Mapa de Cobertura y Uso de la Tierra, Mapa de Tipo de Suelos y Modelo Digital de Elevación de la Cuenca del Río Guarinó Mapa 1:100,000 Bogotá. ideam, 2007, Mapa de Ubicación de Estaciones Meteorológicas de la Cuenca del Río Guarinó Mapa 1:100,000 Bogotá. 